\title{
INTERFACE PROBLEMS IN ELASTO-VISCOPLASTICITY
}

\author{
BY \\ CARSTEN CARSTENSEN (Dept. of Mathematics, Heriot-Watt University, Edinburgh, UK) \\ AND
}

ERNST P. STEPHAN (Institut für Angewandte Mathematik, UNI Hannover, FRG)

\begin{abstract}
This paper is concerned with three-dimensional interface (or transmission) problems in solid mechanics that consist of time-dependent nonlinear problems in a bounded Lipschitz domain and the homogeneous linear elasticity problem in an unbounded exterior domain. The exterior part of the interface problem is rewritten with integral operators on the interface boundary using the Poincaré-Steklov operator. This coupling approach uses the Calderón projector.

We show existence and uniqueness of solutions for three models in elasto-viscoplasticity, namely Gröger's model, Maxwell material, and material of the generalized Burger type. Finally, we sketch corresponding numerical approximation procedures that are a coupling of finite elements and boundary elements in space and difference schemes in time.
\end{abstract}

1. Introduction. This paper gives a uniform approach to the coupling of timedependent nonlinear problems in a bounded Lipschitz domain $\Omega \subseteq \mathbb{R}^{3}$ with a timeindependent problem from linear homogeneous elasticity in an exterior domain $\Omega_{2} \subseteq$ $\mathbb{R}^{3} \backslash \bar{\Omega}$. Three material laws are under consideration describing the interior problem in $\Omega$; they model creeping and relaxation, i.e., the evolution in time of bituminous cements, concrete, ductile metals, polymers, etc. (cf. also [26, p. 353] for an analogy to electrical circuits). We refer to the appendix for further explanations concerning the three models.

i) Gröger's material is given as the system (16)-(19) of partial differential equations and a first-order evolution inclusion (compare Appendix). Eliminating variables this system can be reduced to a single first-order evolution inclusion

$$
b^{\prime} \in \mathscr{A}^{-1} q^{\prime}+\partial \varphi(q),
$$

with prime denoting the time derivative. Now, due to the main theorem on first-order evolution inclusions [25] we obtain existence and uniqueness for the solution of (1), and thus we can show the unique solvability of (16)-(19) by simple resubstitution.

Received December 21, 1992.

1991 Mathematics Subject Classification. Primary 65N30, 65R20, 73C50.

(C)1995 Brown University 
ii) Although the material of the generalized Burger type is of second order in time, it can be rewritten (with a suitable vector $U$ ) as a first-order evolution inclusion

$$
U^{\prime}+\mathscr{A} U \ni F \text {. }
$$

Since $\mathscr{A}$ is maximal monotone we directly conclude existence and uniqueness of a solution of (2) (under suitable initial conditions).

iii) For the classical Maxwell material (in contrast to the previous examples) one cannot substitute one of the variables from the equilibrium condition to apply the main theorem on first-order evolution inclusions. Instead we use a regularization technique (see $\S 6$ ).

In the interface problems we have the conditions of the respective interior problems (i, ii, iii), the homogeneous Navier-Lamé equations for the exterior problem $\Delta^{*} u_{2}=0, u_{2}$ being the displacements with $u_{2}(x) \rightarrow 0,|x| \rightarrow \infty$, and the interface conditions, namely continuity of displacements and tractions on the interface $\Gamma=\bar{\Omega} \cap \bar{\Omega}_{2}$.

We rewrite the exterior problem with boundary integral operators based on the fundamental solution for the Lamé system. Using the Poincaré-Steklov operator the monotonicity property of the nonlinear part carries over to the rewritten form of the interface problems. Thus, the ideas of the proofs-based more or less on the main theorem on evolution inclusions-can be successfully applied proving existence and uniqueness of solutions of the various interface problems.

Since the coupling approach is the same in all three examples, the exterior problem is stated and rewritten in terms of boundary integral operators concerning the Poincaré-Steklov operator in $\S 2$. Some notations for the time-dependent problems and the main theorem on first-order evolution inclusions are recalled in $\S 3$. Then, the three material laws in elasto-viscoplasticity are considered in $\Omega$ whereas the exterior part always describes linear elasticity. In $\S 4$ we analyze Gröger's material $[16,26]$, in $\S 5$ we consider the material of the Burger type [22], and in $\S 6$ we treat the classical Maxwell material [22]. In all cases we show that the interface problem can be rewritten in a form allowing a proof of existence and uniqueness of solutions as well as their numerical approximation. The numerical schemes are based on a coupling of finite elements and boundary elements together with difference approximations in time.

2. The exterior problem. Let $\Omega_{0} \subset \Omega_{1} \subset \mathbb{R}^{3}$ be bounded Lipschitz domains in three dimensions such that $\Omega_{0}$ lies compactly in $\Omega_{1}$. Then, $\Omega:=\Omega_{1} \backslash \bar{\Omega}_{0}$ is the interior domain and $\Omega_{2}:=\mathbb{R}^{3} \backslash \bar{\Omega}_{1}$ is the exterior domain.

The boundary of $\Omega$ is divided into two parts, namely the interior boundary $\Gamma_{0}:=$ $\partial \Omega_{0}$ and the interface $\Gamma:=\partial \Omega_{1}$; cf. Fig. 1. We consider Dirichlet, Neumann, or mixed boundary conditions on $\Gamma_{0}$ and allow the case $\Gamma_{0}=\varnothing$ (whence $\Omega_{0}=\varnothing$ ).

The exterior problem is the homogeneous Lamé system of linear elasticity $[11,12]$

$$
\Delta^{*} u:=-\mu_{2} \Delta u-\left(\lambda_{2}+\mu_{2}\right) \operatorname{grad} \operatorname{div} u=0 \quad \text { in } \Omega_{2}
$$

with $\Delta=$ div grad denoting the Laplace operator, $\mu_{2}, \lambda_{2}$ being the positive Lamé constants. 


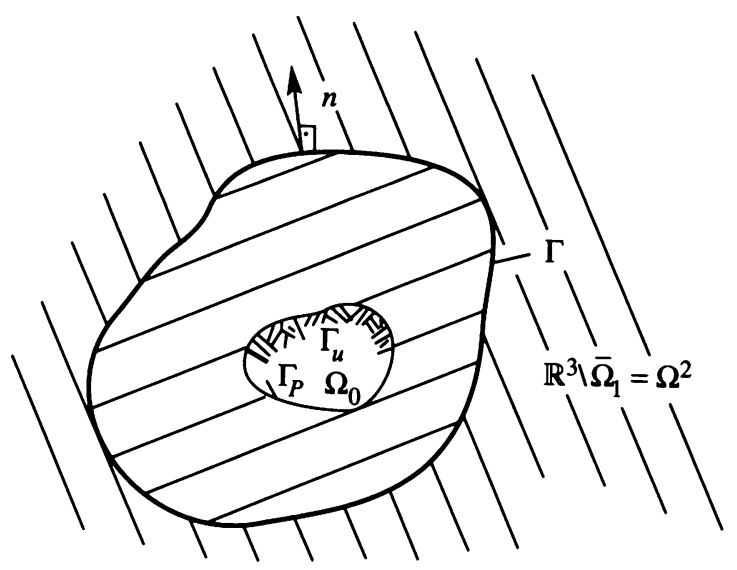

FIG. 1.

Given a smooth vector field $u_{2}$, its Cauchy data on the boundary $\Gamma$ are

$$
\left(\left.u_{2}\right|_{\Gamma},\left.T_{2}\left(u_{2}\right)\right|_{\Gamma}\right)
$$

where $T_{2}\left(u_{2}\right)$ is the conormal derivative defined by

$$
T_{2}\left(u_{2}\right):=2 \mu_{2} \partial_{n} u_{2}+\lambda_{2} n \operatorname{div} u_{2}+\mu_{2} n \times \operatorname{curl} u_{2} .
$$

$\partial_{n}$ denotes the normal derivative, $n$ being the unit normal pointing into $\Omega_{2}$, cf. Fig. 1.

Due to the trace lemma, $\left.u_{2}\right|_{\Gamma} \in H^{1 / 2}\left(\Gamma ; \mathbb{R}^{3}\right)$ whenever $u_{2} \in H_{\mathrm{loc}}^{1}\left(\Omega_{2} ; \mathbb{R}^{3}\right)$, $H_{\text {loc }}^{1}\left(\Omega_{2} ; \mathbb{R}^{3}\right)$ denoting the displacements of locally finite energy.

As e.g. in $[10,11,12]$ the traction $\left.T_{2}\left(u_{2}\right)\right|_{\Gamma}$ can be defined via the First Green formula.

In order to do this, we introduce the following notation:

$$
a_{i j k l}:=\lambda_{2} \delta_{i j} \delta_{k l}+\mu_{2}\left(\delta_{i k} \delta_{j l}+\delta_{i l} \delta_{j k}\right),
$$

$\delta_{i j}=1$ for $i=j$ and $\delta_{i j}=0$ for $i \neq j$. The strain tensor $\varepsilon(u)$ is defined by

$$
\varepsilon_{i j}(u):=\frac{1}{2}\left(u_{i, j}+u_{j, i}\right),
$$

$\left(u_{i, j}\right):=\left(u_{i, j}\right)_{i, j=1,2,3}:=\operatorname{grad} u$. The brackets $\langle\cdot, \cdot\rangle$ always denote the duality between $H^{1 / 2}:=H^{1 / 2}\left(\Gamma ; \mathbb{R}^{3}\right)$ and $H^{-1 / 2}:=H^{-1 / 2}\left(\Gamma ; \mathbb{R}^{3}\right)=\left(H^{1 / 2}\right)^{*}$ such that for $v \in H^{1 / 2}\left(\Gamma ; \mathbb{R}^{3}\right)$ and $w \in L^{2}\left(\Gamma ; \mathbb{R}^{3}\right)$

$$
\langle w, v\rangle=\int_{\Gamma} w \cdot v d \Gamma
$$

Lemma 1 ([12]). Let $u_{2} \in H_{\text {loc }}^{1}\left(\Omega_{2}, \mathbb{R}^{3}\right)$ with $\Delta^{*} u_{2} \in L_{\text {loc }}^{2}\left(\Omega_{2}, \mathbb{R}^{3}\right)$. Then $\left.T_{2}\left(u_{2}\right)\right|_{\Gamma} \in$ $H^{-1 / 2}\left(\Gamma, \mathbb{R}^{3}\right)$ is defined by

$$
\int_{\Omega_{2}} v \Delta^{*} u_{2} d \Omega_{2}=\left\langle T_{2} u_{2},\left.v\right|_{\Gamma}\right\rangle+\Phi_{2}\left(u_{2}, v\right)
$$


for any $v \in H^{1}\left(\Omega_{2} ; \mathbb{R}^{3}\right)$ with compact support and

$$
\Phi_{2}\left(u_{2}, v\right)=\int_{\Omega_{2}} \sum_{i j k l=1}^{3} a_{i j k l} \varepsilon_{k l}\left(u_{2}\right) \varepsilon_{i j}(v) d \Omega_{2} .
$$

According to Lemma 1 the Cauchy data of a function $u_{2} \in H_{\mathrm{loc}}^{1}\left(\Omega_{2}, \mathbb{R}^{n}\right)$ with $\Delta^{*} u_{2}=0$ satisfy

$$
\left(\left.u_{2}\right|_{\Gamma},\left.T_{2}\left(u_{2}\right)\right|_{\Gamma}\right) \in H^{1 / 2} \times H^{-1 / 2}:=H^{1 / 2}\left(\Gamma ; \mathbb{R}^{3}\right) \times H^{-1 / 2}\left(\Gamma ; \mathbb{R}^{3}\right) .
$$

Note that in Eq. (5), $v$ must have a compact support. In order to allow $v \in$ $H^{1}\left(\Omega_{2} ; \mathbb{R}^{3}\right)$, a boundary condition at infinity is required. Following, e.g., $[10,11$, $12,14,18,20]$, we consider solutions that are regular at infinity, which means in the three-dimensional case that $u_{2}$ satisfies Sommerfeld's radiation condition

$$
u_{2}=O\left(\frac{1}{|x|}\right) \text { as }|x| \rightarrow \infty .
$$

Definition 1. Given $(v, t) \in H^{1 / 2} \times H^{-1 / 2}$ the exterior problem consists of finding $u_{2} \in \mathscr{L}_{2}$,

$$
\mathscr{L}_{2}:=\left\{u_{2} \in H_{\mathrm{loc}}^{1}\left(\Omega_{2} ; \mathbb{R}^{3}\right): u_{2} \text { satisfies }(6) \text { and } \Delta^{*} u_{2}=0\right\},
$$

with $\left(\left.u_{2}\right|_{\Gamma}, T_{2} u_{2}\right)=(v, t)$.

For the Lamé operator the fundamental solution $G_{2}$ with kernel $G_{2}(x, y)$-called the Kelvin matrix-is well known,

$$
G_{2}(x, y)=\frac{\lambda_{2}+3 \mu_{2}}{8 \pi \mu_{2}\left(\lambda_{2}+2 \mu_{2}\right)}\left\{\frac{1}{|x-y|} I+\frac{\lambda_{2}+\mu_{2}}{\lambda_{2}+3 \mu_{2}} \frac{(x-y)(x-y)^{\mathrm{T}}}{|x-y|^{3}}\right\} .
$$

$I$ is the unit matrix in $\mathbb{R}^{3}$ and ${ }^{\mathrm{T}}$ denotes the transposed matrix. Since $G$ is analytic in $\mathbb{R}^{3} \times \mathbb{R}^{3}$ without the diagonal we may define its traction

$$
T_{2}(x, y):=T_{2, y}\left(G_{2}(x, y)\right)^{\mathrm{T}}, \quad x \neq y .
$$

Due to the second Green formula, see Lemma 1, the following Somigliana representation formula for $x \in \Omega_{2}$

$$
u_{2}(x)=\left\langle T_{2}(x, \cdot), v\right\rangle-\left\langle G_{2}(x, \cdot), \phi\right\rangle
$$

is proved for Lipschitz domains in [7]. Equation (8) holds for all $u_{2} \in H_{\mathrm{loc}}^{1}\left(\Omega_{2}\right)$ with compact support satisfying (3) and $v=\left.u_{2}\right|_{\Gamma}, \phi=\left.T_{2}\left(u_{2}\right)\right|_{\Gamma}$.

For any $x \in \Omega_{2},(8)$ can be differentiated, giving a representation formula for the stresses $T_{2}\left(u_{2}\right)$. By using the classical jump relations for $x \rightarrow \Gamma$ and inserting the Cauchy data into these formulas, one obtains on $\Gamma$

$$
\left(\begin{array}{l}
v \\
\phi
\end{array}\right)=\mathscr{C}_{2} \cdot\left(\begin{array}{l}
v \\
\phi
\end{array}\right)
$$

where the Calderón projector

$$
\mathscr{C}_{2}=\left(\begin{array}{cc}
\frac{1}{2}+\Lambda_{2} & -V_{2} \\
-D_{2} & \frac{1}{2}-\Lambda_{2}^{\prime}
\end{array}\right)
$$


is defined by means of

$$
\begin{aligned}
& \left(V_{2} \phi\right)(x)=\left\langle G_{2}(x, \cdot), \phi\right\rangle, \\
& \left(\Lambda_{2} v\right)(x)=\left\langle T_{2}(x, \cdot), v\right\rangle, \\
& \left(D_{2} v\right)(x)=-T_{2, x}\left(\left\langle T_{2}(x, \cdot), v\right\rangle\right), \\
& \left(\Lambda_{2}^{\prime} \phi\right)(x)=T_{2, x}\left(\left\langle G_{2}(x, \cdot), \phi\right\rangle\right) \quad(x \in \Gamma) .
\end{aligned}
$$

$V_{2}$ is the single layer potential, $\Lambda_{2}$ is the double layer potential with its dual $\Lambda_{2}^{\prime}$, and $D_{2}$ is the hypersingular operator.

The next lemma recalls some properties of the above operators, namely their domain, continuity, and ellipticity from $[6,7,12]$; see also $[9,10]$. For real Banach spaces $X$ and $Y$ let $\mathscr{L}(X, Y)$ denote the real vector space of bounded linear mappings from $X$ into $Y$.

Lemma $2([7,12])$. Set $H^{1 / 2}:=H^{1 / 2}\left(\Gamma, \mathbb{R}^{3}\right), H^{-1 / 2}:=H^{-1 / 2}\left(\Gamma, \mathbb{R}^{3}\right)$. Then

$$
\begin{aligned}
& V_{2} \in \mathscr{L}\left(H^{-1 / 2} ; H^{1 / 2}\right), \\
& \Lambda_{2} \in \mathscr{L}\left(H^{1 / 2} ; H^{1 / 2}\right), \\
& \Lambda_{2}^{\prime} \in \mathscr{L}\left(H^{-1 / 2} ; H^{-1 / 2}\right), \\
& D_{2} \in \mathscr{L}\left(H^{1 / 2} ; H^{-1 / 2}\right) .
\end{aligned}
$$

$D_{2}$ is positive semidefinite and $V_{2}$ is positive definite, i.e., there exists a constant $\gamma_{2}>0$ such that for all $v \in H^{1 / 2}$ and all $\phi \in H^{-1 / 2}$, it follows that

$$
\left\langle D_{2} v, v\right\rangle \geq 0 \text { and }\left\langle\phi, V_{2} \phi\right\rangle \geq \gamma_{2}\|\phi\|_{H^{-1 / 2}}^{2} .
$$

$D_{2}$ and $V_{2}$ are symmetric, and $\Lambda^{\prime}$ is the dual of $\Lambda$.

The relations between the Calderón projector $\mathscr{C}_{2} \in \mathscr{L}\left(H^{1 / 2} \times H^{-1 / 2} ; H^{1 / 2} \times H^{-1 / 2}\right)$ and the Cauchy data of a function in $\mathscr{L}_{2}$ are recalled in the next lemma.

LEMMA 3 ([11, 12]).

(i) If $u_{2} \in \mathscr{L}_{2}$ then (8) holds for $v:=\left.u_{2}\right|_{\Gamma} \in H^{1 / 2}:=H^{1 / 2}\left(\Gamma, \mathbb{R}^{3}\right)$ and $\phi:=$ $\left.T_{2}\left(u_{2}\right)\right|_{\Gamma} \in H^{-1 / 2}:=H^{-1 / 2}\left(\Gamma, \mathbb{R}^{3}\right)$.

(ii) For any $v \in H^{1 / 2}$ and $\phi \in H^{-1 / 2}$ the vector field $u_{2}$ defined via (8) belongs to $\mathscr{L}_{2}$.

(iii) For $(v, \phi) \in H^{1 / 2} \times H^{-1 / 2}$ the following statements (a) and (b) are equivalent.

(a) $(v, \phi)$ are Cauchy data of some $u_{2} \in \mathscr{L}_{2}$, i.e., $v=\left.u_{2}\right|_{\Gamma}, \phi=\left.T_{2}\left(u_{2}\right)\right|_{\Gamma}$ for some $u_{2} \in \mathscr{L}_{2}$.

(b) $(v, \phi)$ satisfies $(9)$.

(iv) The Calderón projector is a projection in $H^{1 / 2} \times H^{-1 / 2}$ onto its subspace of Cauchy data of weak solutions in $\mathscr{L}_{2}$, i.e., onto $\left\{\left(\left.u_{2}\right|_{\Gamma},\left.T_{2}\left(u_{2}\right)\right|_{\Gamma}\right): u_{2} \in\right.$ $\left.\mathscr{L}_{2}\right\}$.

We are now in a position to prove the following equivalence result concerning the exterior problem. 
THEOREM 1. For any $(v, t) \in H^{1 / 2} \times H^{-1 / 2}:=H^{1 / 2}\left(\Gamma, \mathbb{R}^{3}\right) \times H^{-1 / 2}\left(\Gamma, \mathbb{R}^{3}\right)$ the exterior problem has a solution $u_{2}$ if and only if

$$
t=-S_{2} v
$$

where

$$
S_{2}:=D_{2}+\left(\frac{1}{2}-\Lambda_{2}^{\prime}\right) V_{2}^{-1}\left(\frac{1}{2}-\Lambda_{2}\right) \in \mathscr{L}\left(H^{1 / 2}, H^{-1 / 2}\right) .
$$

In this case the solution $u_{2}$ of the exterior problem is unique and given by the representation formula (8) with $v$ and $\phi:=V_{2}^{-1}\left(\Lambda_{2}-\frac{1}{2}\right) v$.

Proof. Let $u_{2}$ be a solution of the exterior problem. According to Lemma 3(iii) the Cauchy data $v=\left.u_{2}\right|_{\Gamma}, \phi=\left.T_{2}\left(u_{2}\right)\right|_{\Gamma}=t$ satisfy (9) which is equivalent to

$$
V_{2} \phi=\left(\Lambda_{2}-\frac{1}{2}\right) v \text { and } \phi=-D_{2} v+\left(\frac{1}{2}-\Lambda_{2}^{\prime}\right) \phi \text {. }
$$

By Lemma 2, $V_{2}$ is invertible such that $(11)$ is equivalent to

$$
\phi=V_{2}^{-1}\left(\Lambda_{2}-\frac{1}{2}\right) v \text { and } \phi=-D_{2} v+\left(\frac{1}{2}-\Lambda_{2}^{\prime}\right) V_{2}^{-1}\left(\Lambda_{2}-\frac{1}{2}\right) v \text {. }
$$

The last equation in (12) is $t=\phi=-S_{2} v$, which proves the first implication of the theorem.

In order to verify the second implication assume that $(v, t) \in H^{1 / 2} \times H^{-1 / 2}$ satisfies $t=-S_{2} v$. According to Lemma 3(ii) we may define a vector field $u_{2} \in \mathscr{L}_{2}$ via (8) with $v$ and $\phi:=V_{2}^{-1}\left(\Lambda_{2}-\frac{1}{2}\right) v$. Using the jump relations for $x \rightarrow \Gamma$ in (8) we obtain (cf. Lemma 3 (iv))

$$
\left(\begin{array}{c}
u_{2} \mid \Gamma \\
\left.T_{2}\left(u_{2}\right)\right|_{\Gamma}
\end{array}\right)=\mathscr{C}_{2}\left(\begin{array}{l}
v \\
\phi
\end{array}\right)=\left(\begin{array}{c}
\frac{1}{2} v+\Lambda_{2} v-V_{2} \phi \\
-D_{2} v+\left(\frac{1}{2}-\Lambda_{2}^{\prime}\right) \phi
\end{array}\right)=\left(\begin{array}{c}
v \\
-S_{2} v
\end{array}\right)
$$

where the last equality follows from the definitions of $\phi$ and $S_{2}$. Therefore, and since $t=-S_{2} v$, one obtains $\left.u_{2}\right|_{\Gamma}=v,\left.T_{2}\left(u_{2}\right)\right|_{\Gamma}=t$, i.e., $u_{2}$ solves the exterior problem.

It remains to prove uniqueness of the solution $u_{2}$. Assuming the existence of two solutions of the exterior problem with respect to the same Cauchy data $(v, t)$, let $w_{2} \in \mathscr{L}_{2}$ denote their difference. According to Lemma 3(i) we have for $x \in \Omega_{2}$

$$
w_{2}(x)=\left\langle T_{2}(x, \cdot),\left.w_{2}\right|_{\Gamma}\right\rangle-\left\langle G_{2}(x, \cdot),\left.T_{2}(w)\right|_{\Gamma}\right\rangle .
$$

On the other hand, by construction of $w_{2}$, the Cauchy data of $w_{2}$ are homogeneous, i.e., $\left.w_{2}\right|_{\Gamma}=0$ and $\left.T_{2}\left(w_{2}\right)\right|_{\Gamma}=0$, which gives $w_{2}(x)=0$ in (13). Thus, two solutions of the exterior problem with respect to the same Cauchy data are equal.

The following property of the Poincare-Steklov operator is crucial for our boundary conditions at $\Gamma_{0}$. For clarity we present the proof of the following lemma.

Lemma $4([4,8]) . S_{2}$ is positive definite, i.e., there exists $c>0$ such that for any $v \in H^{1 / 2}$ it follows that

$$
\left\langle S_{2} v, v\right\rangle \geq c\|v\|_{H^{1 / 2}}
$$

Proof. According to Lemma $2, S_{2} \in \mathscr{L}\left(H^{1 / 2} ; H^{-1 / 2}\right)$-as defined in (10)-is symmetric and positive semidefinite, i.e., for all $v, w \in H^{1 / 2}$,

$$
\left\langle S_{2} v, w\right\rangle=\left\langle S_{2} w, v\right\rangle \text { and }\left\langle S_{2} v, v\right\rangle \geq 0
$$


hold. With [12, Lemma 4.5] we have that $S_{2}$ differs from a positive-definite operator by a compact perturbation (see also [7]). Thus, since $S_{2}$ is positive semidefinite, it remains to prove that $S_{2} v=0$ for $v \in H^{1 / 2}$ implies $v=0$.

Given $v \in H^{1 / 2}$ with $0=S_{2} v$ we define $u_{2} \in \mathscr{L}_{2}$ by (8) for $\phi=0$. Note that $S_{2} v=0$ implies $D_{2} v=0$ and $\left(\Lambda_{2}-\frac{1}{2}\right) v=0$ (due to the bijectivity of $V_{2}$ from $H^{-1 / 2}$ onto $H^{1 / 2}$ ). Then, as seen in the second part of the proof of Theorem 1, we have $\left.u_{2}\right|_{\Gamma}=v$ and $\left.T_{2}\left(u_{2}\right)\right|_{\Gamma}=\phi=0$. As in the proof of [12, Lemma 4.5], (6) allows us to apply the First Green's formula to $u_{2} \cdot \Delta^{*} u_{2}=0$ in $\Omega_{2}$, which (cf. (5)) leads to

$$
0=\Phi_{2}\left(u_{2}, u_{2}\right)
$$

i.e., $u_{2}$ is a rigid body motion. Because of $(6)$, this gives $u_{2}=0$.

Remark 1. Define $\varepsilon \in \mathscr{L}(H ; L)$ through (4) and let $S:=\gamma^{*} S_{2} \gamma \in \mathscr{L}\left(H ; H^{*}\right)$ with the trace operator $\gamma \in \mathscr{L}\left(H ; H^{1 / 2}\right)$ and its dual $\gamma^{*} \in \mathscr{L}\left(H^{-1 / 2} ; H^{*}\right)$.

Then, according to Korn's inequality, $\varepsilon^{*} \varepsilon+S \in \mathscr{L}\left(H ; H^{*}\right)$ is positive definite [4, Lemma 5].

The proof is based on the fact that $\varepsilon^{*} \varepsilon$ is positive semidefinite, strongly elliptic, and its nullspace $\operatorname{ker}\left(\varepsilon^{*} \varepsilon\right)$ consists of rigid body motions. Thus $\varepsilon^{*} \varepsilon+S$ is injective proving that $\varepsilon^{*} \varepsilon+S$ is positive definite.

3. Notation. If $X$ is a real separable Hilbert space, $\langle\cdot, \cdot\rangle_{X}$ denotes the scalar product in $X$. If $T>0$ is a fixed real number and $1 \leq p \leq \infty$ then let $L^{p}(0, T ; X)$ denote the space of all measurable functions $h:[0, T] \rightarrow X \quad(h$ is measurable if it is the pointwise limit of a sequence of step functions) such that

$$
\|h\|_{L^{p}(0, T ; X)}^{p}:=\int_{0}^{T}\|h(t)\|_{X}^{p} d t<\infty
$$

if $p<\infty$ and with the natural modification for $p=\infty . W_{p}^{1}(0, T ; X)$ denotes the space of all $h \in L^{p}(0, T ; X)$ such that $h$ has a weak derivative $h^{\prime} \in L^{p}(0, T ; X)$. Set

$$
\|h\|_{W_{1}^{p}(0, T ; X)}:=\|h\|_{L^{p}(0, T ; X)}+\left\|h^{\prime}\right\|_{L^{p}(0, T ; X)} .
$$

According to the main theorem on calculus,

$$
v(t)=v(0)+\int_{0}^{t} u(t) d t
$$

whenever $v^{\prime}=u \in L^{1}(0, T ; X)$, we see that $u \in W_{1}^{1}(0, T ; X)$ is absolutely continuous (after changing $u$ on a set of measure zero). Hence

$$
W_{p}^{1}(0, T ; X)=\left\{h \in C(0, T ; X) \mid h^{\prime} \in L^{p}(0, T ; X)\right\}
$$

and $C^{1}(0, T ; X) \subseteq W_{p}^{1}(0, T ; X) \subseteq C(0, T ; X)$ with continuous embeddings.

We recall that a mapping $A: X \rightarrow 2^{X}$ is called multivalued, i.e., $A(x)$ is a subset (possibly empty) of $X$ for any $x \in X$, and $2^{X}$ denotes the set of all subsets of $X . \operatorname{Dom}(A):=\{x \in X \mid A(x) \neq \varnothing\}$ is called the effective domain of a multivalued 
operator $A$. We write $(x, y) \in A$ if $y \in A(x)$. For two multivalued operators $A$ and $B$ we write $A \subseteq B$ if for any $(x, y) \in A,(x, y) \in B$ holds.

A multivalued operator $A$ is called monotone if for all $\left(x_{1}, y_{1}\right),\left(x_{2}, y_{2}\right) \in A$

$$
0 \leq\left\langle y_{2}-y_{1}, x_{2}-x_{1}\right\rangle_{X} \text {. }
$$

A monotone multivalued operator $A$ is called maximal monotone if for any monotone multivalued operator $B$ with $A \subseteq B$ we have $A=B$. It is well known that a multivalued monotone operator $A$ is maximal monotone if and only if $R(I+A)=X$, $I$ being the identity in $X$, and $R(A)$ denoting $\bigcup_{x \in X} A(x)$; cf., e.g., [25, Proposition 55.1].

The subdifferential $\partial \varphi$ of a convex, lower semicontinuous and proper functional $\varphi: X \rightarrow(-\infty,+\infty]$ is maximal monotone; see, e.g., [25, Theorem 47.F] for a proof. Recall that $\varphi$ is proper if it is not equal to $\infty$.

The main theorem on first-order evolution inclusions (cf., e.g., [25, Theorem 55.A, Corollary 55.4]) states for a real separable Hilbert space $X$ and a maximal monotone operator $A: X \rightarrow 2^{X}$ that for given data $u_{0} \in \operatorname{Dom}(A)$ and $b \in W_{2}^{1}(0, T ; X)$ there exists a unique function $u \in W_{2}^{1}(0, T ; X)$ with $u(0)=u_{0}$ and

$$
b \in u^{\prime}+A(u) \text { a.e. in }(0, T) .
$$

Moreover, $u \in W_{\infty}^{1}(0, T ; X)$. The assumptions on the data $f \in W_{2}^{1}(0, T ; X)$ and $u_{0} \in \operatorname{Dom}(A)$ can be weakened, yielding weaker regularity of the solution $u$.

In the applications below we use the following consequence of the main theorem.

Corollary 1. Let $X$ be a real separable Hilbert space and let $\varphi: X \rightarrow(-\infty,+\infty]$ be a convex, lower semicontinuous and proper functional and let $A \in \mathscr{L}(X ; X)$ be positive definite and selfadjoint. Let $u_{0} \in \operatorname{Dom}(\varphi):=\{x \in X \mid \varphi(x)<\infty\}$ and let $b \in W_{2}^{1}(0, T ; X)$. Then there exists a unique function $u \in W_{\infty}^{1}(0, T ; X)$ with $u(0)=u_{0}$ such that

$$
b \in A u^{\prime}+\partial \varphi(u) \text { a.e. in }(0, T) .
$$

Proof. For $A$ being the identity $I$ the corollary is included in the main theorem (cf. [25, Theorem 55.A, Corollary 55.4]). The proof for $A \neq I$ follows the lines in [26, p. 357] changing the norm in the Hilbert space $X$.

4. The nonlinear interface problem for elasto-viscoplastic material with linear hardening. In this section elasto-viscoplastic material with linear hardening of the Gröger type is considered in the interior region $\Omega[16 ; 26$, Chapter 66]. Using Theorem 1, the interface problem is rewritten using boundary integral operators. Following the ideas of $[16 ; 26$, Chapter 66] one proves existence and uniqueness of solutions.

The abstract model below describes slow deformation processes in elasto-viscoplasticity. Some physical interpretations are given in [26, Chapter 66]. Besides the equilibrium condition

$$
\varepsilon^{*} \sigma=\gamma^{*} t+f
$$

for body forces $f \in H^{*}, H:=H_{u}^{1}\left(\Omega, \mathbb{R}^{3}\right)=\left\{u \in H^{1}\left(\Omega ; \mathbb{R}^{3}\right):\left.u\right|_{\Gamma_{u}}=0\right\}, \Gamma_{u} \subseteq$ $\Gamma_{0}, f(0)=0$, surface forces $t \in H^{-1 / 2}=H^{-1 / 2}\left(\Gamma, \mathbb{R}^{3}\right)$ and the stress field $\sigma \in$ 
$L:=L^{2}\left(\Omega, \mathbb{R}_{\mathrm{sym}}^{3 \times 3}\right)$, we consider the following stress-strain relation which is more complicated and involves two further internal variables $p, q \in L$. As usual $L$ is identified with its dual space $L=L^{*}$. p is a so-called plastic part of the strain $\varepsilon(u)$,

$$
\sigma=A(\varepsilon(u)-p)
$$

where $A \in \mathscr{L}(L, L)$ is a linear elasticity operator, i.e., $A$ is symmetric and positive definite.

The constitutive relation for $p$ is more complicated and becomes time-dependent:

$$
p^{\prime} \in \partial \varphi(q) \text { a.e. in }(0, T)
$$

where $\varphi: L \rightarrow(-\infty,+\infty]$ is a convex, lower semicontinuous and proper functional with $0 \in \operatorname{Dom}(\varphi),(0, T)$ is the time interval under consideration and $q$ is the second internal variable. In (18) $p^{\prime}$ denotes the time-derivative of $p$ as introduced in the previous section.

The constitutive relation for $q$ is given by

$$
q+(A+B) p=A \varepsilon(u)
$$

where $B \in \mathscr{L}(L, L)$ is symmetric and positive definite.

For convenient notations, we assume homogeneous initial values. Then, the interface problem for Gröger's material described by (16)-(19) reads as follows.

Definition 2. Given $f \in W_{2}^{2}\left(0, T ; H^{*}\right)$ with $f(0)=0$ the interface problem of this section consists of finding functions

$$
\left(u, u_{2}, \sigma, p, q, t\right) \in W_{2}^{1}\left(0, T ; H \times \mathscr{L}_{2} \times L \times L \times L \times H^{-1 / 2}\right)
$$

satisfying (16), (17), (18), (19), and the initial conditions $(u, \sigma, p, q, t)(0)=0$ as well as the interface conditions

$$
\gamma u=\left.u_{2}\right|_{\Gamma} \in W_{2}^{1}\left(0, T ; H^{1 / 2}\right) \text { and } t=\left.T_{2}\left(u_{2}\right)\right|_{\Gamma} \in W_{2}^{1}\left(0, T ; H^{-1 / 2}\right) .
$$

REMARK 2. The physical background of the time-dependent nonlinear problem can be found in [26, Chapter 66]. There, it is shown that plastic, viscoplastic and elasto-viscoplastic material behavior with linear hardening is included as a particular case. We emphasize that due to the analogy between strain and voltage, stress and current, the stress-strain relation and Ohm's law, we can also apply the analysis of the interface problems at hand in electrical models.

Next we rewrite the interface problem in terms of boundary integral operators (for the notation compare Theorem 1).

TheOREM 2. $\left(u, u_{2}, \sigma, p, q, t\right) \in W_{2}^{1}\left(0, T ; H \times \mathscr{L}_{2} \times L \times L \times L \times H^{-1 / 2}\right)$ solves the interface problem of Def. 2 if and only if $(u, p, q) \in W_{2}^{1}(0, T ; H \times L \times L)$ satisfies a.e. in $(0, T)$

$$
\begin{aligned}
\varepsilon^{*}(q+B p)+S u & =f, \\
q+(A+B) p & =A \varepsilon(u), \\
p^{\prime} & \in \partial \varphi(q),
\end{aligned}
$$

and the initial conditions $(u, p, q)(0)=0$. 
In the latter case, $\sigma$ is given by (17) and $u_{2}$ is given by the representation formula with Cauchy data $(v, t):=\left(\gamma u,-S_{2} \gamma u\right)$.

Proof. Assume that $\left(u, u_{2}, \sigma, p, q, t\right) \in W_{2}^{1}\left(0, T ; H \times \mathscr{L}_{2} \times L \times L \times L \times H^{-1 / 2}\right)$ solves the interface problem of Def. 2. Then, the initial conditions $(u, \sigma, p, q)(0)=$ 0 and (22) as well as (23) are satisfied. Substitution of $\sigma$ from (17) in (16) gives

$$
\varepsilon^{*} A \varepsilon(u)-\gamma^{*} t=f+\varepsilon^{*} A p .
$$

On the other hand, according to the interface conditions and Theorem 1 we have $t=-S_{2} \gamma u$. Taking $t=-S_{2} \gamma u$ in (24) and substituting $\varepsilon^{*} A \varepsilon(u)$ from (19) proves (21).

Conversely, assume that $(u, p, q) \in W_{2}^{1}\left(0, T ; H \times \mathscr{L}_{2} \times L \times L\right)$ satisfy (21), (22), and (23) a.e. in $(0, T)$ as well as the initial conditions $(u, p, q)(0)=0$. Then define $t:=-S_{2} \gamma u \in W_{2}^{1}\left(0, T ; H^{-1 / 2}\right)$ and $v:=\gamma u \in W_{2}^{1}\left(0, T ; H^{1 / 2}\right)$. According to Theorem 1 the representation formula gives a function $u_{2} \in \mathscr{L}_{2}$ having Cauchy data $(v, t)$ a.e. in $[0, T]$. Note that the representation formula is not time-dependent. Therefore $u_{2}$ has the same regularity in time as the data, i.e., $u_{2} \in W_{2}^{1}\left(0, T ; \mathscr{L}_{2}\right)$. Define $\sigma$ by means of (17). Altogether we have $\left(u, u_{2}, \sigma, p, q ; t\right) \in W_{2}^{1}(0, T ; H \times$ $\mathscr{L}_{2} \times L \times L \times L \times H^{-1 / 2}$ ) solving the interface problem of Definition 2 . Note that (22), (23) coincide with (19), (18), respectively, and (17) is satisfied by construction. Furthermore, substituting $\gamma t:=-S u$ in (21) yields

$$
\varepsilon^{*}(q+B p)=f+\gamma^{*} t,
$$

which implies (16) using (22) in connection with (17).

REMARK 3. Note that the assertions of Theorem 2 hold also in the subspace $W_{\infty}^{1}\left(0, T ; H \times \mathscr{L}_{2} \times L \times L \times H^{-1 / 2}\right)$ of $W_{2}^{1}\left(0, T ; H \times \mathscr{L}_{2} \times L \times L \times H^{-1 / 2}\right)$.

Using the equivalent form of Theorem 2 one proves existence and uniqueness of a solution of the interface problem.

THEOREM 3. The interface problem of Definition 2 has a unique solution in $W_{\infty}^{1}(0, T$; $H \times \mathscr{L}_{2} \times L \times L \times L \times H^{-1 / 2}$.

Proof. According to Remark 3 following Theorem 2 it suffices to prove that there exists $(u, p, q) \in W_{\infty}^{1}(0, T ; H \times L \times L)$ satisfying the homogeneous initial conditions as well as $(21),(22)$, and $(23)$ a.e. in $(0, T)$.

We start with the proof of uniqueness and assume that $(u, p, q) \in W_{2}^{1}(0, T ; H \times$ $L \times L)$ exists with the above properties.

Application of $\varepsilon^{*}$ to (22) shows

$$
\varepsilon^{*}(q+B p)+\varepsilon^{*} A p=\varepsilon^{*} A \varepsilon(u) .
$$

Combining this with (21) gives

$$
\left(\varepsilon^{*} A \varepsilon+S\right)(u)=f+\varepsilon^{*} A p .
$$

Define

$$
\widehat{A}:=\varepsilon^{*} A \varepsilon+S \in \mathscr{L}\left(H, H^{*}\right)
$$


and note that $\widehat{A}$ is symmetric and positive definite (compare Remark 1). Due to the Lax-Milgram lemma, $\widehat{A}$ is invertible and (25) is equivalent to

$$
u=\widehat{A}^{-1} f+\widehat{A}^{-1} \varepsilon^{*} A p .
$$

Substitution of (27) into (22) gives

$$
q=A \varepsilon \widehat{A}^{-1} f-\left(A+B-A \varepsilon \widehat{A}^{-1} \varepsilon^{*} A\right) p .
$$

Let

$$
\mathscr{A}:=A+B-A \varepsilon \widehat{A}^{-1} \varepsilon^{*} A \in \mathscr{L}(L, L) .
$$

Using the relations

$$
\begin{aligned}
\left\langle\left(\begin{array}{cc}
\varepsilon^{*} A \varepsilon+S & \varepsilon^{*} A \\
A \varepsilon & A
\end{array}\right)\right. & \left.\left(\begin{array}{l}
u \\
p
\end{array}\right),\left(\begin{array}{l}
u \\
p
\end{array}\right)\right\rangle_{H \times L} \\
& =\langle S u, u\rangle_{H}+\langle A(\varepsilon(u)+p),(\varepsilon(u)+p)\rangle_{L} \geq 0
\end{aligned}
$$

and

$$
\begin{aligned}
\left(\begin{array}{cc}
I & 0 \\
-A \varepsilon\left(\varepsilon^{*} A \varepsilon+S\right)^{-1} & I
\end{array}\right)\left(\begin{array}{cc}
\varepsilon^{*} A \varepsilon+S & \varepsilon^{*} A \\
A \varepsilon & A
\end{array}\right)\left(\begin{array}{cc}
I & -\left(\varepsilon^{*} A \varepsilon+S\right)^{-1} \varepsilon^{*} A \\
0 & I
\end{array}\right) & =\left(\begin{array}{cc}
\varepsilon^{*} A \varepsilon+S & 0 \\
0 & \mathscr{A}-B
\end{array}\right)
\end{aligned}
$$

( 0 and $I$ denote zero and identity in different spaces) one concludes that $\mathscr{A}$ is positive definite due to the corresponding property of $B$. Hence, due to the LaxMilgram lemma, $\mathscr{A}$ is invertible and (28) is equivalent to

$$
p=g-\mathscr{A}^{-1} q \in W_{2}^{1}(0, T ; L)
$$

where we used $g:=\mathscr{A}^{-1} A \varepsilon \widehat{A}^{-1} f \in W_{2}^{2}(0, T ; L)$. Substitution of (30) in (23) shows

$$
g^{\prime} \in \mathscr{A}^{-1} q^{\prime}+\partial \varphi(q)
$$

a.e. in $(0, T)$. Note that $g^{\prime} \in W_{2}^{1}(0, T ; L)$ such that we may apply Corollary 1 to conclude that there exists a unique function $q \in W_{\infty}^{1}(0, T ; L)$ satisfying $(31)$ and $q(0)=0 \in \operatorname{Dom}(\varphi)$. As seen above, any solution $(u, p, q)$ of Theorem 2 satisfies (31) which-together with the initial conditions-determines $q$ uniquely. Then (30) determines $p$ uniquely and finally (27) determines $u$ uniquely.

Secondly, we conclude that the above problem has a solution. As mentioned, (31) together with the initial condition $q(0)=0$ has a solution $q \in W_{\infty}^{1}(0, T ; L)$. Then define $p$ by means of (30) and $u$ by (27). It remains to prove that $(u, p, q) \in$ $W_{\infty}^{1}(0, T ; H \times L \times L)$ solves (21)-(23). According to (30) and (31) we have

$$
p^{\prime}=g^{\prime}-\mathscr{A}^{-1} q^{\prime} \in \partial \varphi(q),
$$

which proves (23). Equation (30) is equivalent to

$$
q+(A+B) p=A \varepsilon \widehat{A}^{-1} \varepsilon^{*} A p+A \varepsilon \widehat{A}^{-1} f .
$$


Using (27) to compute $A \varepsilon(u)$ we get

$$
A \varepsilon(u)=A \varepsilon \hat{A}^{-1} \varepsilon^{*} A p+A \varepsilon \hat{A}^{-1} f .
$$

Then, (32) and (33) prove (22). Finally, (27) leads to

$$
\varepsilon^{*} A \varepsilon(u)+S u=f+\varepsilon^{*} A p .
$$

Since we have already proved (22) we may substitute $A \varepsilon(u)$ from $(22)$ in (34) to prove (21).

We finally sketch the numerical approximation of the interface problem consisting of a Galerkin procedure in space and the implicit Euler method in time. Unfortunately, since $V_{2}^{-1}$ is not known explicitly, the stiffness matrix $\left\langle S u_{i}, u_{j}\right\rangle$ of the Poincaré-Steklov operator $S$ cannot directly be computed numerically and hence has to be approximated leading to the coupling of finite elements and boundary elements.

Definition 3. Let $\left(\mathscr{T}_{h} \mid h \in I\right)$ be a family of regular triangulations of the polygonal domain $\Omega ; h \in I \subseteq(0, \infty), 0 \in \bar{I}$, may be regarded as the maximal mesh size. If $P_{k}\left(\mathbb{R}^{m}\right)$ denotes the polynomials of degree $\leq k$ with values in $\mathbb{R}^{m}$ then let

$$
\begin{aligned}
H_{h} & :=\left\{u \in C\left(\Omega ; \mathbb{R}^{3}\right):\left.u\right|_{\Gamma_{u}}=0, \forall T \in \mathscr{T}_{h},\left.u\right|_{T} \in P_{1}\left(\mathbb{R}^{3}\right)\right\} \subseteq H, \\
H_{h}^{-1 / 2} & :=\left\{v \in L^{\infty}\left(\Gamma ; \mathbb{R}^{3}\right): \forall T \in \mathscr{T}_{h},\left.v\right|_{\bar{T} \cap \Gamma} \in P_{0}\left(\mathbb{R}^{3}\right)\right\} \subseteq H^{-1 / 2}, \\
L_{h} & :=\left\{\sigma \in L^{\infty}(\Omega): \forall T \in \mathscr{T}_{h},\left.\sigma\right|_{T} \in P_{0}\left(\mathbb{R}_{\mathrm{sym}}^{3 \times 3}\right)\right\} \subseteq L,
\end{aligned}
$$

$H_{h}\left(H_{h}^{-1 / 2}, L_{h}\right)$ being piecewise linear (constant) trial functions in $H\left(H^{1 / 2}, L\right)$ with respect to the regular triangulation $\mathscr{T}_{h}$.

Define $\widetilde{S}_{h} \in \mathscr{L}\left(H_{h} ; H_{h}^{*}\right)$ through $\left\langle\widetilde{S}_{h} u_{h}, v_{h}\right\rangle_{H}$ for $u_{h}, v_{h} \in H_{h}$ as follows. Given $u_{h} \in H_{h}$ solve the Galerkin equations: find $\phi_{h} \in H_{h}^{-1 / 2}$ with

$$
\left\langle\psi_{h}, V_{2} \phi_{h}\right\rangle=\left\langle\psi_{h},\left(\frac{1}{2}-\Lambda_{2}\right) \gamma u_{h}\right\rangle \quad \text { (for all } \psi_{h} \in H_{h}^{-1 / 2} \text { ) }
$$

(note that $V_{2}$ is positive definite) and set for arbitrary $v_{h} \in H_{h}$

$$
\left.\left\langle\widetilde{S}_{2} u_{h}, v_{h}\right\rangle_{H}:=\left\langle D_{2} \gamma u_{h}+\frac{1}{2}-\Lambda^{\prime}\right) \phi_{h}, v_{h}\right\rangle_{H} .
$$

The discrete problem for (21)-(23) is now written as follows. Set $\left(u_{h, 0}, \sigma_{0, h}, q_{h, 0}\right)$ $:=0$ and compute $\left(u_{h, j}, \sigma_{h, j}, q_{h, j}\right) \in H_{h} \times L_{h} \times L_{h}$ for $j=1,2,3, \ldots, n$, recursively, with

$$
\begin{aligned}
\left\langle q_{h, j}+B p_{h, j}, \varepsilon\left(v_{h}\right)\right\rangle_{L}+\left\langle\tilde{S}_{h} u_{h, j}, v_{h}\right\rangle & =\left\langle f\left(\frac{j T}{n}\right), v_{h}\right\rangle_{H}, \\
\left\langle q_{h, j}+(A+B) p_{h, j}, r_{h}\right\rangle_{L} & =\left\langle A \varepsilon\left(u_{h, j}\right), r_{h}\right\rangle_{L}, \\
\left\langle\frac{p_{h, j}-p_{h, j-1}}{T / n}, q_{h, j}-s_{h}\right\rangle_{L} & \leq \varphi\left(q_{h, j}\right)-\varphi\left(s_{h}\right),
\end{aligned}
$$

for all $v_{h} \in H_{h}, r_{h} \in L_{h}, s_{h} \in L_{h}$.

REMARK 4. As is shown in [4], $\widetilde{S}_{2}$ approximates $S$; cf. also [12] where-for another class of materials-the equation for $\phi_{h}$ is explicitly added to the discrete system. 
We conclude this section with a regard to the semidiscrete time discretization [16] where we have above $H_{h} \times H_{h}^{-1 / 2} \times L_{h} \times L_{h}=H \times H^{-1 / 2} \times L \times L$. Define $\left(u_{n}, q_{n}, p_{n}\right) \in$ $W_{2}^{1}(0, T ; H \times L \times L)$ by linear interpolation of $\left(u_{h, j}, q_{h, j}, p_{h, j}\right)_{j=0, \ldots, n}$ where the interpolation points are $(j \cdot T / n)_{j=0, \ldots, n}$.

COROllary 2. In the semidiscrete case of a time discretization it follows that

$$
\lim _{n \rightarrow \infty}\left(u_{n}, q_{n}, p_{n}\right)=(u, q, p) \text { in } W_{2}^{1}(0, T ; H \times L \times L) .
$$

Proof. The proof of [16, Theorem 4.3] for the time discretization (for the nonlinear material behavior of the interior problem only) works verbatim for our interface problem if we replace $\varepsilon^{*} \varepsilon$ with $\varepsilon^{*} \varepsilon+S$.

5. Nonlinear interface problem for viscoelastic material of the Burger type. In this section elasto-viscoplastic material of the Burger type from [22] is considered in the interior region $\Omega$ whereas in the exterior domain we have again linear elastic material. Again using Theorem 1 we represent the exterior problem via boundary integral operators. Therefore, we can show existence and uniqueness of the solution of the resulting interface problem by modifying the analysis for the interior problem.

Some physical interpretations of the abstract model below are given in [22] modeling slow deformation processes in elasto-viscoplasticity. Inertia is included in this model such that we obtain an equilibrium condition of second order in time since we have $v=u^{\prime}$ for the velocity $v$ with the displacements $u$. Incorporating inertia the equilibrium condition (16) is extended to

$$
\rho v^{\prime}=\operatorname{div} \sigma+f \text { in } \Omega
$$

while the boundary conditions are as above, i.e.,

$$
\sigma n=t \text { on } \Gamma \text {, }
$$

and we may have Neumann data on $\Gamma_{0} \mid \bar{\Gamma}_{u}$. The problem is of second order in time since the additional term $\rho v^{\prime}=\rho u^{\prime \prime}$ describes inertia of the material.

Besides the equilibrium conditions for the stresses $\sigma$, we have further constitutive relations and internal variables $\varepsilon_{1}, \varepsilon_{2}$ [22].

The interior problem can be written as the first-order evolution inclusion

$$
(0, f / \rho, 0,0) \in U^{\prime}+\mathscr{A} U \text { a.e. in }[0, T], \quad U(0)=U_{0}
$$

where $U_{0} \in \check{D}(\mathscr{A}),\left(A_{2} \varepsilon_{2}+A_{4} \varepsilon(u)\right) n=t$ on $\Gamma$, and

$$
\mathscr{A} U:=\left(\begin{array}{c}
-v \\
-\frac{1}{\rho} \operatorname{div}\left(A_{2} \varepsilon_{2}+A_{4} \varepsilon(u)\right) \\
\partial \varphi_{3}^{*}\left(A_{2} \varepsilon_{2}\right)+\partial \varphi_{1}^{*}\left(A_{2} \varepsilon_{2}-A_{1} \varepsilon_{1}\right)-\varepsilon(v) \\
-\partial \varphi_{1}^{*}\left(A_{2} \varepsilon_{2}-A_{1} \varepsilon_{1}\right)
\end{array}\right)
$$

for $U=\left(u, v, \varepsilon_{2}, \varepsilon_{1}\right) \in \mathscr{H}:=H \times L^{\prime} \times L \times L$.

The condition $v=u^{\prime}$ is the first condition of (36), noting $\sigma:=\left(A_{2} \varepsilon_{2}+A_{4} \varepsilon(u)\right)$ the equilibrium (35) is the second condition in (36) while the constitutive relations are given in the third and fourth components of (36). The boundary condition $\sigma n=t$ is treated in the domain of $\mathscr{A}$; see below. 
Here, we use the following notation: $f \in W_{2}^{1}\left(0, T ; L^{\prime}\right)$ with $f(0)=0$ is a given body force, $L^{\prime}:=L^{2}\left(\Omega ; \mathbb{R}^{3}\right), t \in W_{2}^{1}\left(0, T ; H^{-1 / 2}\right)$ is a surface force, $H^{-1 / 2}:=$ $H^{-1 / 2}\left(\Gamma, \mathbb{R}^{3}\right), \sigma=\left(A_{2} \varepsilon_{2}+A_{4} \varepsilon(u)\right) \in W_{2}^{1}(0, T ; L)$ is the stress field, and $L:=$ $L^{2}\left(\Omega, \mathbb{R}_{\mathrm{sym}}^{3 \times 3}\right) . \rho \in L^{\infty}(\Omega)$ is a given density with $\rho_{0}>0$ such that

$$
\rho \geq \rho_{0} \text { a.e. in } \Omega \text {. }
$$

$A_{1}, A_{2}, A_{4} \in \mathscr{L}\left(\mathbb{R}_{\mathrm{sym}}^{3 \times 3}, \mathbb{R}_{\text {sym }}^{3 \times 3}\right)$ are symmetric and positive definite; for $j=1,3$,

$$
\varphi_{j}: \mathbb{R}_{\mathrm{sym}}^{3 \times 3} \rightarrow[0, \infty], \quad(\tau) \mapsto \nu_{j}|\tau|^{q_{j}} \quad \text { if } \operatorname{tr}(\tau)=0 \text { and } \infty \text { otherwise, }
$$

where $\operatorname{tr}(\tau):=\sum_{i=1,2,3} \tau_{i i}$ and $q_{j}, \nu_{j}$ are positive constants. $\varphi_{j}^{*}$ denotes the Legendre-Fenchel transform of $\varphi_{j}$, and $\partial \varphi_{j}^{*}$ denotes its subdifferential. For the definition and some properties of the Legendre-Fenchel transform $\varphi_{j}^{*}: L \rightarrow[-\infty, \infty]$,

$$
\varphi_{j}^{*}(\tau)=\sup _{\sigma \in \mathbb{R}_{\mathrm{sym}}^{3 \times 3}}\left(\sigma: \tau-\varphi_{j}(\sigma)\right) \quad(\text { for all } \tau \in L)
$$

with the scalar product in $\mathbb{R}^{3 \times 3}, \sigma: \tau:=\sum_{i, j=1,2,3} \sigma_{i j} \cdot \tau_{j i}$; we refer, e.g., to [13, $22,25]$. The above particular case for $\varphi_{j}$ is a simple example; it is only needed in the following that $\varphi_{j}$ is a positive normal integrand [13] with $\varphi_{j}(0)=0$ in $\Omega$. For convenience of notation, we neglect the possible space dependence of the functionals and remark that the results below also hold in the general case.

Note that $\mathscr{A}$ is a multivalued operator in the Hilbert space

$$
\mathscr{H}:=H \times L^{\prime} \times L \times L
$$

which (provided $\Gamma_{u}$ has positive surface measure) can be endowed with the scalar product

$$
\langle U, V\rangle_{1}:=\left\langle A_{4} \varepsilon\left(u_{1}\right), \varepsilon\left(v_{1}\right)\right\rangle_{L}+\left\langle\rho u_{2}, v_{2}\right\rangle_{L^{\prime}}+\left\langle A_{2} u_{3}, v_{3}\right\rangle_{L}+\left\langle A_{1} u_{4}, v_{4}\right\rangle_{L}
$$

setting $U=\left(u_{1}, \ldots, u_{4}\right)$ and $V=\left(v_{1}, \ldots, v_{4}\right)$. We will consider the following domains of $\mathscr{A}$ :

$$
\begin{aligned}
\check{D}(\mathscr{A}): & =\left\{\left(u, v, \varepsilon_{2}, \varepsilon_{1}\right) \in \mathscr{H} \mid v \in H, \operatorname{div}\left(A_{2} \varepsilon_{2}+A_{4} \varepsilon(u)\right) \in L^{\prime},\right. \\
\left.\partial \varphi_{3}^{*}\left(A_{2} \varepsilon_{2}\right) \cap L \neq \varnothing, \partial \varphi_{1}^{*}\left(A_{2} \varepsilon_{2}-A_{1} \varepsilon_{1}\right) \cap L \neq \varnothing\right\}, & \\
D(\mathscr{A}):= & \left\{\left(u, v, \varepsilon_{2}, \varepsilon_{1}\right) \in \check{D}(\mathscr{A}) \mid\left(A_{2} \varepsilon_{2}+A_{4} \varepsilon(u)\right) n=0 \text { on } \Gamma\right\}, \\
\widehat{D}(\mathscr{A}):= & \left\{\left(u, v, \varepsilon_{2}, \varepsilon_{1}\right) \in \check{D}(\mathscr{A}) \mid\left(A_{2} \varepsilon_{2}+A_{4} \varepsilon(u)\right) n+S_{2} \gamma u=0 \text { on } \Gamma\right\} .
\end{aligned}
$$

$D(\mathscr{A})$ is used in [22] where the inhomogeneous Neumann boundary conditions are taken into account via some substitution.

RemarK 5. According to $\left(u, v, \varepsilon_{2}, \varepsilon_{1}\right) \in \check{D}(\mathscr{A})$, we have $\sigma:=\left(A_{2} \varepsilon_{2}+A_{4} \varepsilon(u)\right) \in$ $L=L^{2}\left(\Omega ; \mathbb{R}_{\text {sym }}^{3 \times 3}\right)$ and $\operatorname{div} \sigma \in L^{\prime}=L^{2}\left(\Omega ; \mathbb{R}^{3}\right)$. Thus, we may define $\sigma n$ by means of Green's formula

$$
\int_{\Gamma} w \sigma n d \Gamma=\int_{\Omega}(\varepsilon(w): \sigma+w \operatorname{div} \sigma) d \Omega
$$


for any $w \in H$. According to the trace lemma, $\gamma w \in H^{1 / 2}$ can be extended to a function $w \in H$. Thus (40) shows $\sigma n \in H^{-1 / 2}$ such that the Neumann boundary condition $\sigma n=t$ on $\partial \Omega$ makes sense.

The interface problem-which consists of (36) in the interior domain $\Omega$ and of the exterior problem given by Definition 1 -reads as follows.

Definition 4. Under the above notation, given $f$, the interface problem of this section consists in finding functions

$$
\left(\left(u, v, \varepsilon_{2}, \varepsilon_{1}\right), u_{2}, t\right) \in W_{2}^{1}\left(0, T ; \check{D}(\mathscr{A}) \times \mathscr{L}_{2} \times H^{-1 / 2}\right)
$$

satisfying (36) as well as the interface conditions

$$
\left(\gamma u,\left(A_{2} \varepsilon_{2}+A_{4} \varepsilon(u)\right) n\right)=\left.\left(u_{2}, T_{2}\left(u_{2}\right)\right)\right|_{\Gamma} \in W_{2}^{1}\left(0, T ; H^{1 / 2} \times H^{-1 / 2}\right) .
$$

Next we rewrite this interface problem with boundary integral operators.

Theorem 4. $\left(U, u_{2}, t\right) \in W_{2}^{1}\left(0, T ; \mathscr{H} \times \mathscr{L}_{2} \times H^{-1 / 2}\right)$ solves the interface problem of Definition 4 if and only if $U \in W_{2}^{1}(0, T ; \mathscr{H})$ satisfies $(36)$ and

$$
U \in \widehat{D}(\mathscr{A}) \text { a.e. in }[0, T] \text {. }
$$

In the latter case, $u_{2}$ is given by the representation formula with Cauchy data $\left(\gamma u,-S_{2} \gamma u\right)$.

Proof. Assume that $\left(U, u_{2}, t\right) \in W_{2}^{1}\left(0, T ; \check{D}(\mathscr{A}) \times \mathscr{L}_{2} \times H^{-1 / 2}\right)$ solves the interface problem of Definition 4. According to Theorem 1 and the interface conditions we have

$$
t=-S_{2} \gamma u=\left(A_{2} \varepsilon_{2}+A_{4} \varepsilon(u)\right) n=\left.T_{2}\left(u_{2}\right)\right|_{\Gamma} \in W_{2}^{1}\left(0, T ; H^{-1 / 2}\right) .
$$

Therefore, $U \in \widehat{D}(\mathscr{A})$ a.e. in $[0, T]$.

Conversely, assume that $U=\left(u, v, \varepsilon_{2}, \varepsilon_{1}\right) \in W_{2}^{1}(0, T ; \mathscr{l})$ satisfies $(36)$ and $U \in \widehat{D}(\mathscr{A})$ a.e. in $[0, T]$. Then, define $t:=-S_{2} \gamma u \in W_{2}^{1}\left(0, T ; H^{-1 / 2}\right)$ and $w:=\gamma u \in W_{2}^{1}\left(0, T ; H^{1 / 2}\right)$. According to Theorem 1 the representation formula gives a function $u_{2} \in \mathscr{L}_{2}$ having Cauchy data $(w, t)$ a.e. in $[0, T]$. Note that the representation formula is not time-dependent. Therefore $u_{2}$ has the same regularity in time as the data, i.e., $u_{2} \in W_{2}^{1}\left(0, T ; \mathscr{L}_{2}\right)$. Since $U \in \widehat{D}(\mathscr{A})$ we have

$$
\left(A_{2} \varepsilon_{2}+A_{4} \varepsilon(u)\right) n=-S_{2} \gamma u=t \text { on } \Gamma,
$$

which shows (41) since $t=T_{2}\left(u_{2}\right)$. Thus $\left(U, u_{2}, t\right) \in W_{2}^{1}\left(0, T ; \check{D}(\mathscr{A}) \times \mathscr{L}_{2} \times\right.$ $\left.H^{-1 / 2}\right)$ solves the interface problem of Definition 4.

Define the scalar product $\langle\cdot, \cdot\rangle_{2}$ on $\mathscr{H}$ as follows:

$$
\langle U, V\rangle_{2}:=\langle U, V\rangle_{1}+\left\langle S u_{1}, v_{1}\right\rangle_{H},
$$

$U=\left(u_{1}, \ldots, u_{4}\right)$, and $V=\left(v_{1}, \ldots, v_{4}\right)$. Since $S=\gamma^{*} S_{2} \gamma \in \mathscr{L}\left(H, H^{*}\right)$ is symmetric and positive semidefinite, but $S_{2} \in \mathscr{L}\left(H^{1 / 2} ; H^{-1 / 2}\right)$ is positive definite, $\langle\cdot, \cdot\rangle_{2}$ is a scalar product of the real Hilbert space $\mathscr{H}$ (compare Remark 1). 
ThEOREM 5. $\mathscr{A}: \widehat{D}(\mathscr{A}) \rightarrow 2^{\mathscr{H}}$ is maximal monotone in the real Hilbert space $\left(\mathscr{H},\langle\cdot, \cdot\rangle_{2}\right)$. Thus there exists a unique solution of $(36)$ as well as of the interface problem from Definition 4.

Proof. It is proved in [22] that $\mathscr{A}: D(\mathscr{A}) \rightarrow 2^{\mathscr{H}}$ is maximal monotone in the real Hilbert space $\left(\mathscr{H},\langle\cdot, \cdot\rangle_{1}\right)$ if $\Gamma_{u}$ has positive surface measure. Following the lines of the proof (in [22, Sec. 3.4]) the above theorem can be proved in the present slightly different situation. Modifications are required just if Green's formula is applied. The examination below is given only for completeness.

Similarly to [22] we prove that $\mathscr{A}: \widehat{D}(\mathscr{A}) \rightarrow 2^{\mathscr{H}}$ is monotone. For $U=$ $\left(u_{1}, \ldots, u_{4}\right), V=\left(v_{1}, \ldots, v_{4}\right) \in \widehat{D}(\mathscr{A})$,

$$
\begin{aligned}
\langle\mathscr{A} U & -\mathscr{A} V, U-V\rangle_{2} \\
= & -\left\langle\left(\varepsilon\left(u_{2}-v_{2}\right), A_{4} \varepsilon\left(u_{1}-v_{1}\right)+A_{2}\left(u_{3}-v_{3}\right)\right\rangle_{L}\right. \\
& -\left\langle\operatorname{div}\left(A_{4} \varepsilon\left(u_{1}-v_{1}\right)+A_{2}\left(u_{3}-v_{3}\right)\right), u_{2}-v_{2}\right\rangle_{L^{\prime}} \\
& +\left\langle\partial \varphi_{3}^{*}\left(A_{2} u_{3}\right)-\partial \varphi_{3}^{*}\left(A_{2} v_{3}\right), A_{2}\left(u_{3}-v_{3}\right)\right\rangle_{L} \\
& +\left\langle\partial \varphi_{1}^{*}\left(A_{2} u_{3}-A_{1} u_{4}\right)-\partial \varphi_{1}^{*}\left(A_{2} v_{3}-A_{1} v_{4}\right), A_{2}\left(u_{3}-v_{3}\right)-A_{1}\left(u_{4}-v_{4}\right)\right\rangle_{L} \\
& -\left\langle S\left(u_{2}-v_{2}\right),\left(u_{1}-v_{1}\right)\right\rangle_{H} .
\end{aligned}
$$

Using Green's formula for the first term on the right-hand side and the interface conditions in $\widehat{D}(\mathscr{A})$,

$$
\begin{aligned}
-\left\langle\operatorname{div}\left(A_{4} \varepsilon\left(u_{1}-v_{1}\right)+A_{2}\left(u_{3}-v_{3}\right)\right), u_{2}-v_{2}\right\rangle_{L^{\prime}} \\
=\left\langle A_{4} \varepsilon\left(u_{1}-v_{1}\right), \varepsilon\left(u_{2}-v_{2}\right)\right\rangle_{L^{\prime}}+\left\langle A_{2}\left(u_{3}-v_{3}\right), \varepsilon\left(u_{2}-v_{2}\right)\right\rangle_{L^{\prime}} \\
\quad+\left\langle S\left(u_{1}-v_{1}\right),\left(u_{2}-v_{2}\right)\right\rangle_{H} .
\end{aligned}
$$

Thus, since $\partial \varphi_{1}^{*}, \partial \varphi_{3}^{*}$ are monotone $\left(\varphi_{1}, \varphi_{3}\right.$ are convex), one concludes

$$
\langle\mathscr{A} U-\mathscr{A} V, U-V\rangle_{2} \geq 0,
$$

i.e., $\mathscr{A}: \widehat{D}(\mathscr{A}) \rightarrow 2^{\mathscr{H}}$ is monotone.

In order to prove that $\mathscr{A}: \widehat{D}(\mathscr{A}) \rightarrow 2^{\mathscr{H}}$ is maximal monotone it suffices to show that for any $Z=\left(z_{1}, \ldots, z_{4}\right) \in \mathscr{H}$ and $\tau>0$ there exists some $U=\left(u_{1}, \ldots, u_{4}\right) \in$ $\widehat{D}(\mathscr{A})$ with

$$
\tau U+\mathscr{A} U=Z
$$

(cf., e.g., [25, Proposition 55.1]).

Given $Z=\left(z_{1}, \ldots, z_{4}\right) \in \mathscr{H}$ and $\tau:=\frac{1}{\Delta t}>0$ consider the functional

$$
\widehat{J}: H \rightarrow \mathbb{R} \cup\{\infty\}, \quad w \mapsto J(w)+\frac{1}{2}\langle S w, w\rangle_{H}
$$

where the functional $J: H \rightarrow \mathbb{R} \cup\{\infty\}$ is motivated in [22, Subsection 3.4.3] and defined by

$$
\begin{aligned}
J(w):= & \left.\frac{1}{2}\left\langle\rho\left(\tau w-2 z_{2}\right), w\right\rangle_{L^{\prime}}+\frac{1}{2 \tau}<A_{4} \varepsilon\left(w+2 z_{1}\right), \varepsilon(w)\right\rangle_{L} \\
& +\int_{\Omega} \psi^{*}\left(A_{2}\left(\varepsilon(w)+z_{3}+z_{4}\right)\right) d \Omega
\end{aligned}
$$


where $\psi, \varphi_{\Delta t}: \mathbb{R}_{\text {sym }}^{3 \times 3} \rightarrow \mathbb{R} \cup\{\infty\}$ are defined as in [22, Subsection 3.4.3], i.e.,

$$
\begin{aligned}
\psi(d) & :=\frac{\tau}{2} A_{2} d: d+\tau \varphi_{\Delta t}^{*}\left(A_{2} d\right)+\varphi_{3}^{*}\left(A_{2} d\right), \\
\varphi_{\Delta t}(d) & :=\frac{1}{2} A_{1} d: d+\frac{1}{\tau} \varphi_{1}\left(\tau d-z_{4}\right)
\end{aligned}
$$

for $d \in \mathbb{R}_{\text {sym }}^{3 \times 3}$. $\varphi_{\Delta t}^{*}$ is the Legendre-Fenchel transform of $\varphi_{\Delta t}$, and $\psi^{*}$ is the Legendre-Fenchel transform of $\psi$.

In [22, Subsection 3.4 .4 ] it is proved that $J$ is strictly convex, lower semicontinuous and coercive on $H$. Thus, the same holds for $\widehat{J}$, since due to Remark 1 there exists $c>0$ with

$$
\frac{1}{\tau}\left\langle A_{4} \varepsilon(w), \varepsilon(w)\right\rangle_{L}+\langle S w, w\rangle \geq c\|w\|_{H}^{2}
$$

for all $w \in H$. Therefore, there exists a unique minimizer $u_{2} \in H$ of $\hat{J}$, i.e., $0 \in \partial \widehat{J}\left(u_{2}\right)$.

In [22, Subsection 3.4.5] the subdifferential of $J$ is calculated. Since $\widehat{J}-J$ is convex and continuous, the sum rule gives

$$
\partial \widehat{J}=\partial J+S \text {. }
$$

Define $u_{1}:=\frac{1}{\tau}\left(u_{2}+z_{1}\right) \in H$. Applying the characterization of $0 \in \partial J$ from [22, Subsection 3.4.5] to our case $0 \in \partial \widehat{J}\left(u_{2}\right)$ we obtain some

$$
u_{3} \in \partial\left(\int_{\Omega} \psi^{*}\left(A_{2}\left(\varepsilon\left(u_{2}\right)+z_{3}+z_{4}\right)\right) d \Omega\right)
$$

with

$$
\begin{aligned}
0= & \left\langle\rho\left(\tau u_{2}-z_{2}\right), w\right\rangle_{L^{\prime}}+\left\langle A_{4} \varepsilon\left(u_{1}\right), \varepsilon(w)\right\rangle_{L} \\
& +\left\langle A_{2} \varepsilon(w), u_{3}\right\rangle_{L}+\left\langle S u_{2}, w\right\rangle_{H}
\end{aligned}
$$

for all $w \in H$ and $u_{1}=\frac{1}{\tau}\left(z_{1}+u_{2}\right)$. Using Green's formula again, i.e.,

$$
\begin{aligned}
-\left\langle\operatorname{div}\left(A_{4} \varepsilon\left(u_{1}\right)+A_{2} u_{3}\right),\right. & w\rangle_{L^{\prime}} \\
& =\left\langle A_{4} \varepsilon\left(u_{1}\right)+A_{2} u_{3}, \varepsilon(w)\right\rangle_{L}-\int_{\Gamma} w \cdot\left(A_{4} \varepsilon\left(u_{1}\right)+A_{2} u_{3}\right) n d \Gamma,
\end{aligned}
$$

this leads to

$$
\begin{aligned}
\rho\left(\tau u_{2}-z_{2}\right)-\operatorname{div}\left(A_{4} \varepsilon\left(u_{1}\right)+A_{2} u_{3}\right)=0 & \text { in } \Omega, \\
\left(A_{4} \varepsilon\left(u_{1}\right)+A_{2} u_{3}\right) n+S_{2} \gamma u_{1}=0 & \text { on } \Gamma .
\end{aligned}
$$

Note that (44) is the second component of (43). Note also with $\varepsilon_{2}=u_{3}$ and $u=u_{1}$ that $(45)$ coincides with the interface conditions in $\widehat{D}(\mathscr{A})$. We have already defined $u_{1}, u_{2}, u_{3}$. Since the remaining considerations in the proof do not concern any boundary condition one can argue verbatim as in [22] to obtain $u_{4}$ such that $U=$ $\left(u_{1}, \ldots, u_{4}\right) \in \widehat{D}(\mathscr{A})$ satisfies $(43)$.

This proves that $\mathscr{A}: \widehat{D}(\mathscr{A}) \rightarrow 2^{\mathscr{H}}$ is maximal monotone. Consequently, according to the main theorem on first-order evolution inclusions, Eq. (36) has exactly one solution and therefore, due to the equivalence given by Theorem 4 , the interface problem of Definition 4 has a unique solution. 
Finally, we sketch a numerical approximation using notation from Def. 3. Given a time step $\Delta t:=T / n, n$ being a natural number, setting $\left(u_{h, 0}, v_{h, 0}, \varepsilon_{2, h, 0}, \varepsilon_{1, h, 0}\right)$ $:=0$, compute $U_{h, j}:=\left(u_{h, j}, v_{h, j}, \varepsilon_{2, h, j}, \varepsilon_{1, h, j}\right) \in H_{h} \times H_{h} \times L_{h} \times L_{h}$ for $j=$ $1, \ldots, n$, recursively,

$$
\begin{gathered}
F\left(\frac{j T}{n}\right) \in \frac{U_{h, j}-U_{h, j-1}}{T / n}+\mathscr{A}\left(U_{h, j}\right), \\
\left(A_{2} \varepsilon_{2, h, j}+A_{4} \varepsilon\left(u_{h, j}\right)\right) n+\widetilde{S}_{2, h} \gamma u_{h, j}=0,
\end{gathered}
$$

where $S_{2, h} \gamma$ is defined by $\gamma^{*} S_{2, h} \gamma=\widetilde{S}_{h}$.

The discrete problem leads to the problem (43) and then to the minimization of $\widehat{J}$ (where $S$ is replaced with $\widetilde{S}_{h}$ ) yielding existence and uniqueness of the solution of the discrete problem.

REMARK 6. In [22] this full discretization is considered for the purely interior problem only and, by numerical examples, the reliability of this algorithm is shown. There, instead of $H_{h}$, the use of further trial functions for the approximation of divergence-free velocity fields is discussed [22, Sec. 4.6].

6. Maxwell material. In this section we treat the classical Maxwell material [22] in the form

$$
\varepsilon\left(u^{\prime}\right)-A \sigma^{\prime}=k \cdot \sigma^{D}
$$

where $0 \leq k \in L^{\infty}(\Omega ; \mathbb{R})$ and

$$
\sigma^{D}:=\sigma-\frac{1}{3} \operatorname{tr} \sigma I, \quad \operatorname{tr} \sigma:=\sum_{j=1,2,3} \sigma_{j j}
$$

and $I \in \mathbb{R}^{3 \times 3}$ is the unit matrix. $A \in \mathscr{L}(L ; L)$ is symmetric and positive definite. In addition to the constitutive relation we provide the equilibrium condition

$$
\varepsilon^{*} \sigma=\gamma^{*} t+f
$$

for body forces $f \in H^{*}, H:=H_{u}^{1}\left(\Omega, \mathbb{R}^{3}\right), f(0)=0$, surface forces $t \in H^{-1 / 2}=$ $H^{-1 / 2}\left(\Gamma, \mathbb{R}^{3}\right)$ and the stress field $\sigma \in L:=L^{2}\left(\Omega, \mathbb{R}_{\text {sym }}^{3 \times 3}\right)$.

The interface problem reads as follows.

Definition 5. Given $f \in W_{2}^{2}\left(0, T ; H^{*}\right)$ with $f(0)=0$, the interface problem of this section consists of finding functions

$$
\left(u, u_{2}, \sigma, t\right) \in W_{2}^{1}\left(0, T ; H \times \mathscr{L}_{2} \times L \times H^{-1 / 2}\right)
$$

satisfying (46), (47) and the initial conditions $(u, \sigma, t)(0)=0$ as well as the interface conditions

$$
\gamma u=\left.u_{2}\right|_{\Gamma} \in W_{2}^{1}\left(0, T ; H^{1 / 2}\right) \text { and } t=\left.T_{2}\left(u_{2}\right)\right|_{\Gamma} \in W_{2}^{1}\left(0, T ; H^{-1 / 2}\right) .
$$

As in the previous sections we rewrite the interface problem in terms of boundary integral operators; the proof is omitted. 
THEOREM 6. $\left(u, u_{2}, \sigma, t\right) \in W_{2}^{1}\left(0, T ; H \times \mathscr{L}_{2} \times L \times H^{-1 / 2}\right)$ solves the interface problem of Definition 5 if and only if $(u, \sigma) \in W_{2}^{1}(0, T ; H \times L)$ satisfies (46) a.e. in $(0, T)$ and

$$
\varepsilon^{*} \sigma+S u=f
$$

and homogeneous initial conditions on $(u, \sigma)$.

In the latter case, $u_{2}$ is given by the representation formula with Cauchy data $(v, t):=\left(\gamma u,-S_{2} \gamma u\right)$.

THEOREM 7. The interface problem of Definition 5 as well as the problem of Theorem 6 have unique solutions.

Proof. We apply a regularization technique to (46), (49) and consider for given $\nu>0$ the auxiliary problem $\left(P_{\nu}\right)$ : Find $\left(u_{\nu}, \sigma_{\nu}\right) \in W_{2}^{1}(0, T ; H \times L)$ such that $\left(u_{\nu}, \sigma_{\nu}\right)(0)=0$ and

$$
\begin{aligned}
\varepsilon^{*} \sigma_{\nu}+\left(\nu \varepsilon^{*} \varepsilon+S\right)\left(u_{\nu}\right) & =f, \\
\varepsilon\left(u_{\nu}^{\prime}\right)-A \sigma_{\nu}^{\prime} & =k \cdot \sigma_{\nu}^{D} .
\end{aligned}
$$

Since $\left(\nu \varepsilon^{*} \varepsilon+S\right) \in \mathscr{L}\left(H ; H^{*}\right)$ is positive definite, (50) is equivalent to

$$
u_{\nu}=\left(\nu \varepsilon^{*} \varepsilon+S\right)^{-1}\left(f-\varepsilon^{*} \sigma_{\nu}\right) .
$$

Substitution of $u_{\nu}$ in (51) leads to a first-order evolution equation which has a unique solution $\sigma_{\nu}$ (cf. Corollary 1). According to (52), this yields a unique solution $\left(u_{\nu}, \sigma_{\nu}\right)$ of problem $\left(P_{\nu}\right)$.

Consider a function $\chi \in W_{2}^{2}(0, T ; L)$ with $\chi(0)=0$ and

$$
f=\varepsilon^{*} \chi
$$

and define

$$
\tau_{\nu}:=\chi-\sigma_{\nu} \in W_{2}^{1}(0, T ; L) .
$$

The function $\chi$ can be determined by solving

$$
\varepsilon^{*} \varepsilon(v)=f \in \Omega,\left.\quad v\right|_{\partial \Omega}=0
$$

and letting $\chi=\varepsilon(v)$ for instance. Substitution of $\sigma_{\nu}=\chi-\tau_{\nu}$ and $u_{\nu}$ from (52) in (51) leads to

$$
\begin{aligned}
\int_{0}^{T}\left\langle A_{\nu} \tau_{\nu}^{\prime}, \tau_{\nu}^{\prime}\right\rangle_{L} d s=\int_{0}^{T}\left\langle k \chi^{D}, \tau_{\nu}^{\prime}\right\rangle_{L} d s \\
\quad+\int_{0}^{T}\left\langle A \chi^{\prime}, \tau_{\nu}^{\prime}\right\rangle_{L} d s-\left\langle\frac{k}{2} \tau_{\nu}^{D}(T) ; \tau_{\nu}^{D}(T)\right\rangle_{L}
\end{aligned}
$$

where $A_{\nu}:=A+\varepsilon\left(S+\nu \varepsilon^{*} \varepsilon\right)^{-1} \varepsilon^{*}$. Since $A$ is positive definite, (53) leads to a constant $c>0, c$ does not depend on $\nu$, such that

$$
\left\|\tau_{\nu}^{\prime}\right\|_{L^{2}(0, T ; L)} \leq c
$$

i.e., $\left(\tau_{\nu}\right)_{\nu>0}$ is uniformly bounded in $W_{2}^{1}(0, T ; L)$ and thus the same holds for $\left(\sigma_{\nu}\right)_{\nu>0}$. Hence, according to (50) and (51), we have with $S=\gamma^{*} S_{2} \gamma$ that $\left(S_{2} \gamma u_{\nu}^{\prime}\right)_{\nu>0}$ 
is uniformly bounded in $L^{2}\left(0, T ; H^{-1 / 2}\right)$ and $\left(\varepsilon\left(u_{\nu}^{\prime}\right)\right)_{\nu>0}$ is uniformly bounded in $L^{2}(0, T ; L)$, respectively. Hence, $\left(\left(\varepsilon^{*} \varepsilon+S\right) u_{\nu}\right)_{\nu>0}$ is uniformly bounded in $W_{2}^{1}\left(0, T ; H^{*}\right)$. Therefore, Remark 1 yields that $\left(u_{\nu}\right)_{\nu}>0$ is uniformly bounded in $W_{2}^{1}(0, T ; H)$.

Since $\left(u_{\nu}, \sigma_{\nu}\right)_{\nu>0}$ is a bounded family in $W_{2}^{1}(0, T ; H \times L)$ the Banach-Alaoglu theorem [21] shows that there exists some parameter sequence $\left(\nu_{n}\right)$ with $\lim _{n \rightarrow \infty} \nu_{n}$ $=0$ such that $\left(u_{\nu_{n}}, \sigma_{\nu_{n}}\right)$ converges weakly toward some $(u, \sigma)$ in $W_{2}^{1}(0, T ; H \times L)$. It is a straightforward calculation that $(u, \sigma)$ then solves the problem of Theorem 6.

It remains to prove the uniqueness of a solution of (46), (49) satisfying homogeneous initial values. In order to do this it suffices to prove $(u, \sigma)=0$ whenever $(u, \sigma)$ solves this problem for $f=0$. Multiplication of (46) with $\sigma^{\prime}$, integration on $(0, T)$, using the main theorem on calculus and $\varepsilon^{*} \sigma^{\prime}=-S u^{\prime}$ lead to

$$
-\left(\int_{0}^{T}\left(\left\langle S u^{\prime}, u^{\prime}\right\rangle_{H}+\left\langle A \sigma^{\prime}, \sigma^{\prime}\right\rangle_{L}\right) d t=\left\langle\frac{k}{2} \sigma^{D}(T), \sigma^{D}(T)\right\rangle_{L} \geq 0 .\right.
$$

Since $S_{2}$ and $A$ are positive definite and $S=\gamma^{*} S_{2} \gamma$, this proves $\sigma=0$ and $\gamma u=0$. Hence, from (46) we have $\varepsilon(u)=0$ so that $\left(\varepsilon^{*} \varepsilon+S\right) u=0$ gives $u=0$ due to Remark 1.

Finally, we sketch the numerical approximation of the solutions of (46), (49) using notation from Definition 3. The discrete problem-consisting of coupling finite elements and boundary elements in space with the implicit Euler method in timereads as follows. Setting $\left(u_{h, 0}, \sigma_{h, j}\right):=0$ compute $\left(u_{h, j}, \sigma_{h, j}\right) \in H_{h} \times L_{h}$ for $j=1,2,3, \ldots, n$, recursively, with

$$
\begin{aligned}
\left\langle\sigma_{h, j}, \varepsilon\left(v_{h}\right)\right\rangle_{L}+\left\langle\widetilde{S}_{h} u_{h, j}, v_{h}\right\rangle & =\left\langle f\left(\frac{j T}{n}\right), v_{h}\right\rangle_{H}, \\
\left\langle\frac{\varepsilon\left(u_{h, j}\right)-\varepsilon\left(u_{h, j-1}\right)}{T / n}-A \frac{\sigma_{h, j}-\sigma_{h, j-1}}{T / n}, \tau_{h, j}\right\rangle_{L} & =\left\langle\sigma_{h, j}^{D}, \tau_{h, j}\right\rangle_{L}
\end{aligned}
$$

for all $v_{h} \in H_{h}, \tau_{h} \in L_{h}$.

Using the regularization technique from the proof of Theorem 7 one can prove that the discrete problem has a unique solution. The numerical analysis of the above algorithm is included in [5] proving convergence of this procedure.

Appendix. Here we consider the three models of this paper. In Fig. 2 springs (denoted by $A, B, A_{1}, A_{2}, A_{4}$ ) describe elastic material components such that stress $\sigma$ results from strain $\varepsilon$ in a linear manner, e.g., $\sigma=A^{-1} \varepsilon$. The dissipative elements (denoted by $\varphi, \varphi_{1}, \varphi_{3}$ ) describe viscous or perfect plastic behavior, i.e., the time derivative of the strain, which is called the strain rate, depends on the stress as in the case of a Newtonian fluid: $\varepsilon^{\prime} \in \partial \varphi(\sigma)$. This form includes the normal rule in plasticity giving the Prandtl-Reuß model. For particular cases of dissipative functionals see $[22,26]$.

The equations corresponding to Fig. 2 can be obtained as follows. For any element $j$ introduce variables $\varepsilon_{j}$ and $\sigma_{j}$ for strain and stress and write down the law of the 


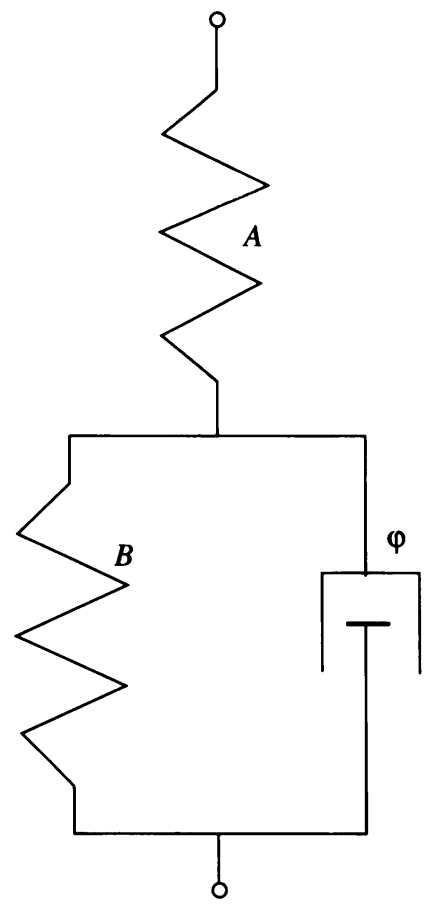

(a)

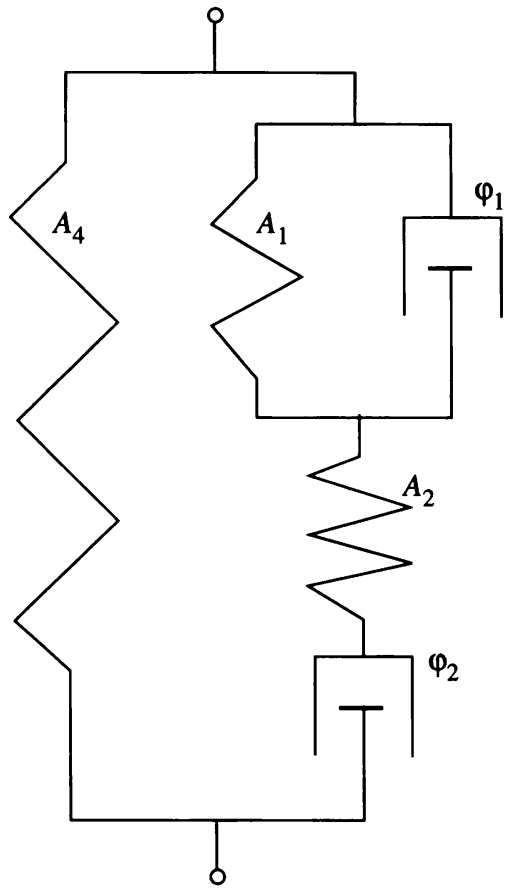

(b)

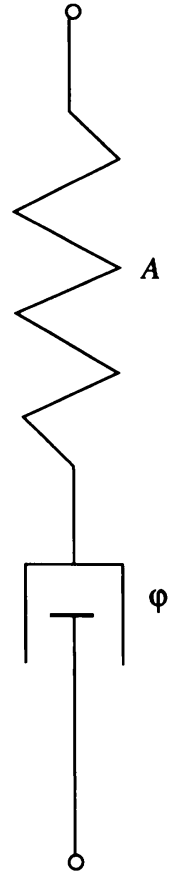

(c)

FIG. 2.

element. Then, calculate the relations between the introduced variables. If two elements are serial (i.e., they build a line) the stresses are equal and the total strain is the sum of the two strains. If two elements are parallel the strains are equal and the total stress is the sum of the two stresses. Using the above rules calculate the final relation between total stress (always denoted by $\sigma$ ) and total strains $\varepsilon(u)$ where $u$ is the displacement of the element.

i) The rheological model for Gröger's material is shown in Fig. 2a. If $\varepsilon_{1}, \varepsilon_{2}, \varepsilon_{3}$ and $\sigma_{1}, \sigma_{2}, \sigma_{3}$ denote the strain and the stress of the elements denoted as $A, B$, $\varphi$, respectively, we have

$$
\sigma_{1}=A \varepsilon_{1}, \quad \sigma_{2}=B \varepsilon_{2}, \quad \varepsilon_{3}^{\prime} \in \partial \varphi\left(\sigma_{3}\right) .
$$

Since $B$ and $\varphi$ are parallel,

$$
\varepsilon_{2}=\varepsilon_{3}, \quad \sigma:=\sigma_{2}+\sigma_{3} .
$$

Since $A$ and $(B, \varphi)$ are serial

$$
\sigma_{1}=\sigma, \quad \varepsilon(u)=\varepsilon_{1}+\varepsilon_{2} .
$$

The weak form of the (quasi-static) equilibrium condition $\varepsilon^{*} \sigma+\gamma^{*} t=f$ can be obtained from the strong form

$$
f+\operatorname{div} \sigma=0 \quad \text { in } \Omega, \quad \sigma n=t \quad \text { on } \Gamma
$$

by multiplication with a test function from $H$, integration over $\Omega$, and using Green's formula with any Dirichlet and Neumann boundary condition. 
Letting $p:=\varepsilon_{2}, q:=\sigma_{3}$ gives $\sigma=B p+q$, and the above relations imply

$$
\begin{aligned}
\varepsilon^{*}(B p+q)+\gamma^{*} t & =f, \\
(A+B) p+q & =A \varepsilon(u), \\
p^{\prime} & \in \partial \varphi(q) .
\end{aligned}
$$

ii) The rheological model for material of the Burger type is shown in Fig. 2b. The situation is slightly more complicated than in i). We have

$$
\sigma_{2}=A_{2} \varepsilon_{2}, \quad \varepsilon_{3}^{\prime} \in \varphi_{3}^{*}\left(\sigma_{3}\right), \quad \sigma_{4}=A_{4} \varepsilon_{4}
$$

and we write

$$
\sigma_{1}^{e}=A_{1} \varepsilon_{1}, \quad \sigma_{1}^{p} \in \varphi_{1}\left(\varepsilon_{1}^{\prime}\right)
$$

for the stresses corresponding to $A_{1}$ and $\varphi_{1}$, respectively, $\varepsilon_{1}$ for the strains; $\varphi_{j}^{*}$ : $L \rightarrow[-\infty, \infty]$ is the Legendre-Fenchel transform of $\varphi_{j}$ defined by means of

$$
\varphi_{j}^{*}(\tau)=\sup _{\sigma \in \mathbb{R}_{\mathrm{sym}}^{3 \times 3}}\left(\sigma: \tau-\varphi_{j}(\sigma)\right) \quad(\text { for all } \tau \in L)
$$

( : being the scalar product in $\mathbb{R}^{3 \times 3}$ ). It can be proved that $\sigma_{1}^{p} \in \varphi_{1}\left(\varepsilon_{1}^{\prime}\right)$ if and only if $\varepsilon_{1}^{\prime} \in \varphi_{1}^{*}\left(\sigma_{1}^{p}\right)[13,22,25]$. Calculating the relations between the elements in Fig. $2 \mathrm{~b}$ we obtain

$$
\sigma=\sigma_{1}+\sigma_{4}, \quad \sigma_{1}:=\sigma_{1}^{e}+\sigma_{1}^{p}=\sigma_{2}=\sigma_{3}
$$

and

$$
\varepsilon(u)=\varepsilon_{4}=\varepsilon_{1}+\varepsilon_{2}+\varepsilon_{3} .
$$

From this one finally concludes

$$
\begin{aligned}
\sigma & =A_{4} \varepsilon(u)+A_{2} \varepsilon_{2}, \\
\varepsilon_{1}^{\prime} & \in \partial \varphi_{1}^{*}\left(A_{2} \varepsilon_{2}-A_{1} \varepsilon_{1}\right), \\
\varepsilon\left(u^{\prime}\right)-\varepsilon_{1}^{\prime}-\varepsilon_{2}^{\prime} & \in \partial \varphi_{3}^{*}\left(A_{2} \varepsilon_{2}\right) .
\end{aligned}
$$

These conditions explain $\sigma=A_{4} \varepsilon(u)+A_{2} \varepsilon_{2}$ used in $\S 5$ and lead with (35) and $u^{\prime}=v$ to the components of (37) in (36).

iii) The rheological model for a material of the Maxwell type is shown in Fig. 2c. Let $\varepsilon_{1}, \varepsilon_{2}$ correspond to $A, \varphi$. As above we get $\varepsilon(u)=\varepsilon_{1}+\varepsilon_{2}$ and $\sigma_{1}=A^{-1} \varepsilon_{1}$ as well as $\varepsilon_{2}^{\prime} \in \varphi\left(\sigma_{2}\right)$. Since $\sigma=\sigma_{1}=\sigma_{2}$ this yields

$$
\varepsilon\left(u^{\prime}\right)-A \sigma^{\prime} \in \varphi(\sigma) .
$$

Setting $\varphi(\sigma):=\frac{k}{2} \sigma^{D}: \sigma^{D}$ we obtain (46).

\section{REFERENCES}

[1] J. Bielak and R. C. MacCamy, An exterior interface problem in two-dimensional elastodynamics, Quart. Appl. Math. 41, 143-159 (1983)

[2] F. Brezzi and C. Johnson, On the coupling of boundary integral and finite element methods, Calcolo 16, 189-201 (1979)

[3] C. Carstensen, Interface problems in solid mechanics, Habilitation thesis, University of Hanover, 1993 
[4] C. Carstensen, Interface problem in holonomic elasto-plasticity, Math. Meth. Appl. Sci. 16, 819-835 (1993)

[5] C. Carstensen, Coupling of FEM and BEM for interface problems in viscoplasticity and plasticity with hardening, SIAM J. Numer. Anal. (1994), accepted for publication

[6] M. Costabel, Symmetry's methods for the coupling of finite elements and boundary elements, In: C. A. Brebia et al. (eds.), Boundary Elements IX, Vol. 1, Springer-Verlag, Berlin, 1987, pp. 411-420

[7] M. Costabel, Boundary integral operators on Lipschitz domains: Elementary results, SIAM J. Math. Anal. 19, 613-626 (1988)

[8] M. Costabel, private communication, 1992

[9] M. Costabel and E. P. Stephan, Boundary integral equations for mixed boundary value problems in polygonal domains and Galerkin approximation, Banach Center Publ. 15, 175-251 (1985)

[10] M. Costabel and E. P. Stephan, A direct boundary integral equation method for transmission problems, J. Math. Anal. Appl. 106, 367-413 (1985)

[11] M. Costabel and E. P. Stephan, Integral equations for transmission problems in linear elasticity, J. Integral Equations Appl. 2, 211-223 (1990)

[12] M. Costabel and E. P. Stephan, Coupling of finite and boundary element methods for an elastoplastic interface problem, SIAM J. Numer. Anal. 27, 1212-1226 (1990)

[13] I. Ekeland and R. Temam, Convex Analysis and Variational Problems, North-Holland, Amsterdam, 1976

[14] G. N. Gatica and G. C. Hsiao, The coupling of boundary element and finite element methods for a nonlinear exterior boundary value problem, Z. Anal. Anwendungen 8, 377-387 (1989)

[15] G. N. Gatica and G. C. Hsiao, On the coupled BEM and FEM for a nonlinear exterior Dirichlet problem in $R^{2}$, Numer. Math. 61, 171-214 (1992)

[16] K. Gröger, Initial-value problems for elastoplastic and elasto-viscoplastic systems (S. Fučik and A. Kufner, eds.), Teubner, Leipzig, 1979

[17] H. Han, A new class of variational formulations for the coupling of finite and boundary element methods, J. Comput. Math. 8, 223-232 (1990)

[18] G. C. Hsiao, E. P. Stephan, and W. L. Wendland, On the Dirichlet problem in elasticity for a domain exterior to an arc, Comput. Appl. Math. 34, 1-19 (1991)

[19] C. Johnson and J. C. Nedelec, On the Coupling of Boundary Integral and Finite Element Methods, Math. Comp. 35 (152), 1063-1079 (1980)

[20] V. D. Kupradze, editor, Three-dimensional Problems of the Mathematical Theory of Elasticity and Thermoelasticity, North-Holland Series in Appl. Math. Mech. 25, North-Holland Publ. Co., Amsterdam, 1979

[21] W. Rudin, Functional Analysis, McGraw-Hill, 1973

[22] P. Le Tallec, Numerical analysis of viscoelastic problems, Recherches en Mathématiques Appliquées 15, Springer-Verlag, Berlin, 1990

[23] W. L. Wendland, On asymptotic error estimates for combined BEM and FEM, in Finite and Boundary Element Techniques from a Mathematical and Engineering Point of View (E. Stein and W. L. Wendland, eds.), CISM Courses 301, Springer-Verlag, New York, 1988, pp. 273-331

[24] E. Zeidler, Nonlinear Functional Analysis and its Applications II, Vols. A and B, Springer-Verlag, New York, 1990

[25] E. Zeidler, Nonlinear Functional Analysis and its Applications III, Springer-Verlag, New York, 1985

[26] E. Zeidler, Nonlinear Functional Analysis and its Applications IV, Springer-Verlag, New York, 1988 